\title{
Equipamento médico-hospitalar: uma gestão na área da saúde
}

\author{
Hospital medical equipment: management in health
}

\author{
Lisiane Marcolin de Almeida', Helena Terezinha Hubert Silva' \\ Universidade Federal de Ciências da Saúde de Porto Alegre, Porto Alegre (RS), Brasil.
}

\section{RESUMO}

Objetivo: O objetivo do artigo é refletir teoricamente acerca da temática: gestão de materiais médico-hospitalares na área saúde. Método: Trata-se de um estudo teórico reflexivo. Resultados: A aquisição de equipamento médico-hospitalares em diferentes âmbitos do setor da saúde exige constantes atualizações devido às mudanças e ao surgimento de novos materiais. A gestão neste aspecto foca-se por um lado à redução de custos, e por outro à priorização da saúde dos clientes. Uma medida que viabiliza a gestão é a aquisição de material de boa qualidade, com bom custo e benefício por meio da autonomia e com a participação ativa dos profissionais que atuam na assistência, na escolha do equipamento e no controle de custos. Conclusões: A avaliação e as análises desenvolvidas neste estudo são válidas por sua contribuição ao entendimento das dificuldades e das limitações existentes no processo de gerenciamento do equipamento médico-hospitalar.

PALAVRAS-CHAVE: Gestão. Equipamento médico-hospitalar. Saúde.

\section{ABSTRACT}

Objective: The objective of this article is to reflect theoretically on the theme: management of medical and hospital materials in the health area. Method: This is a theorical-reflexive study. Results: The acquisition of medical equipment in different areas of the health sector, requires constant updates due to changes and introduction of new products. The management in this aspect is focused on one hand to reduce costs, and on the other hand the prioritization of customers' health. A measure that ensures security to the acquisition of good quality materials with good cost and benefit, is through professional's active participation and freestanding work, assisting the choice of material and therefore costs control. Conclusion: The evaluation and analysis developed in this study are valid for its contribution to the understanding of the existing limitations and difficulties in the medicalhospital supplies management process.

Recebido: Maio 02, 2016 Aceito: Jun. 23, 2016

\section{COMO CITAR ESTE ARTIGO}

Almeida LM, Silva HTH. Equipamento médico-hospitalar: uma gestão na área da saúde. Interdisciplinary Journal of Health Education. 2016 Jan-Jul;1(1):32-39. http://dx.doi. org/10.4322/ijhe2016007

\section{CORRESPONDÊNCIA}

Lisiane Marcolin de Almeida

Universidade Federal de Ciências da Saúde de Porto Alegre

Rua Monsenhor Antônio Guilherme

Grings, 51, apto 801, torre 1, Bairro

Sarandi, CEP 91110-042, Porto Alegre

(RS), Brasil

lisianemarcolin@hotmail.com
KEYWORDS: Management. Medical hospital equipment. Health.

\section{FONTE DE FINANCIAMENTO}

Nenhuma.

\section{CONFLITO DE INTERESSE}

Os autores declararam não

haver conflitos de interesse.

\section{O estudo foi realizado na}

Instituição Universidade Federal

de Ciências da Saúde de Porto

Alegre, Porto Alegre (RS), Brasil.

Todos os autores leram e aprovam

a versão final submetida ao

Interdisciplinary Journal of

Health Education (IJHE). 


\section{Introdução}

O Brasil apresenta um contínuo crescimento com o gasto na saúde, relacionado à universalização do acesso a partir da criação do Sistema Único de Saúde (SUS), ao aumento da expectativa de vida da população e, sobretudo, à incorporação tecnológica das últimas décadas. Esses aspectos contribuem significativamente para o aumento dos custos hospitalares, com reflexo direto na qualidade do serviço e nos recursos públicos e privados disponíveis para seu financiamento ${ }^{1}$.

Um fator importante para aumento dos custos na saúde em todos os países são as novas despesas relacionadas à tecnologia ${ }^{2}$. A aquisição de equipamentos médico-hospitalares, em diferentes âmbitos do setor da saúde, exige constantes atualizações devido às mudanças e aos surgimentos de novos produtos. A gestão, nesse aspecto, foca-se por um lado à redução de custos e, por outro, à priorização da saúde dos clientes. Entretanto, o efeito da tecnologia sobre os custos da saúde pode ser analisado de maneira positiva na redução de despesas e torna as práticas profissionais eficientes e mais precisas ${ }^{3,4}$.

No Brasil, a regulação do mercado de dispositivos médicos é realizada pela Agencia Nacional de Vigilância Sanitária (ANVISA) e, dessa forma, os produtos devem estar registrados na entidade antes da sua comercialização ${ }^{5}$.

AANVISA define produtos para saúde como "[...] produtos utilizados na realização de procedimentos médicos, odontológicos e fisioterápicos, bem como no diagnóstico, tratamento, na reabilitação ou monitoração de pacientes [...]". Dentre os produtos para saúde, encontram-se os equipamentos e materiais de saúde

[...] que são aparelhos, materiais ou acessórios cujo uso ou aplicação esteja ligado à defesa e proteção da saúde individual ou coletiva, à higiene pessoal ou de ambientes, ou a fins diagnósticos e analíticos, os cosméticos e perfumes, e, ainda, os produtos dietéticos, ópticos, de acústica médica, odontológicos e veterinários $[\ldots]^{5}$.

A Rede Brasileira de Avaliação de Tecnologias em Saúde (REBRATS) criada pela Portaria $\mathrm{n}^{\mathrm{o}} 2.915$, de 12 de dezembro de 2011, busca estabelecer a ponte entre pesquisa, política e gestão, fornecendo subsídios para decisões de incorporação, monitoramento e abandono de tecnologias. A REBRATS prima por promover e por difundir a avaliação de tecnologias em saúde no Brasil e no âmbito internacional e entende essa análise como um processo abrangente por meio do qual são verificados os impactos clínicos, sociais e econômicos das tecnologias em saúde, levando-se em consideração, entre outros aspectos, a eficácia, efetividade, segurança, custos e custo-efetividade ${ }^{6}$.

A instituição hospitalar possui uma gestão diferenciada de outras organizações pelo fato de apresentar características próprias como, por exemplo, a atuação ininterrupta, o fato de lidar com clientes em momentos de maior fragilidade, exigindo um aporte assistencial holístico, multidisciplinaridade de profissionais que nelas atuam, recursos tecnológicos disponíveis e eficientes, a fim de ofertar maior segurança tanto para paciente quanto para profissional. Todas essas características influenciam no custo da instituição ${ }^{7}$.

Custos hospitalares são gastos relativos a equipamentos e serviços utilizados na produção médica do hospital. O custo total dos produtos e serviços de uma organização hospitalar é baseado no preço do serviço ou produto, número de unidades e intensidade do serviço. O custo total da hospitalização de um paciente está relacionado ao tempo de internação, à unidade de internação - centro de tratamento intensivo (CTI) ou unidade clínica e cirúrgica, à quantidade de recursos utilizados e ao preço nos diferentes níveis de atendimento ${ }^{2}$. Para a Associação Nacional de Hospitais Privados (ANAHP), outro fator que contribui para elevação do custo nos hospitais é a carga tributária brasileira sobre o preço final dos equipamentos de saúde, visto que mais de $80,0 \%$ dos dispositivos médicos são importados 8 .

Os gastos com materiais nas instituições hospitalares representam aproximadamente de $15,0 \%$ a $25,0 \%$ das despesas, tanto nas instituições públicas, como nas privadas ${ }^{9}$. 
Indo ao encontro dessa informação, os dados do observatório da ANAHP apontam que a segunda maior despesa dos hospitais compreende os insumos hospitalares, totalizando $24,5 \%$ do custo no ano de $2014^{8}$. Na mesma perspectiva, analisou-se a evolução do gasto com internações para um plano de saúde com abrangência no estado de São Paulo, durante um período de cinco anos. Esse estudo apontou que os materiais respondem pelas parcelas mais expressivas do gasto hospitalar, correspondendo a uma elevação de custo médio de $118,7 \%$, o equivalente a $\mathrm{R} \$ \mathrm{R} \$ 2.823,47$ por internação ${ }^{10}$. Contudo, por outro lado, o estudo conduzido em uma Unidade de Terapia Intensiva Neonatal (UTI Neonatal), num Hospital Universitário do Paraná, nas 100 horas de observação, foram constatados gastos por uso indevido de material, num total de $\mathrm{R} \$ 7.208,95$, caracterizando também um grande impacto no orçamento hospitalar ${ }^{11}$.

A gestão de materiais, dentro de uma organização hospitalar, é uma unidade fundamental de apoio à redução de custos e prejuízos, composta pelos setores de licitações de compras, serviço de controle de qualidade e almoxarifado, visando atender às demandas institucionais por recursos materiais, bens e serviços ${ }^{12}$.

Nesse contexto, o planejamento e a gestão de custos hospitalares viabilizam a redução de gastos e o desperdício de insumos por meio de escolhas de alternativas possíveis, com o objetivo de oferecer qualidade máxima ao menor custo ${ }^{12}$. Entretanto esse gerenciamento de materiais depende do envolvimento dos profissionais diretamente ligados às áreas onde ocorre o efetivo consumo, de forma a garantir a usabilidade adequada com mínimo de prejuízo ${ }^{7}$.

Ao considerar as afirmações descritas e a análise do cenário atual, envolvendo a gestão de material médico-hospitalar, o estudo justifica-se como relevante e essencial para o desenvolvimento técnico-científico dos profissionais e gestores de instituições de saúde, visto que é notória a ausência de envolvimento desses profissionais assistenciais no gerenciamento de insumos. O despertar inicial do pesquisador na área surgiu a partir da observação da prática diária nos serviços de saúde.

O objetivo do artigo é refletir teoricamente acerca da temática: gestão de materiais médico-hospitalares na área saúde.

Trata-se de um estudo teórico de natureza reflexiva que utiliza embasamento no universo teórico já elaborado ${ }^{13}$. Para a elaboração do presente trabalho, as seguintes etapas foram percorridas: estabelecimento da questão norteadora, objetivo da pesquisa e, após, leitura e reflexão sobre o tema.

Para guiar a pesquisa, formulou-se a seguinte questão: Como é realizado o gerenciamento do material médico-hospitalar nas organizações de saúde?

O estudo foi construído com base nas leituras críticas de publicações de gerenciamento de equipamentos médico-hospitalares nas instituições de saúde. Foram utilizadas, também, outras fontes de informação como livros, manuais, teses e dissertações, além de documentos oficiais do Ministério da Saúde.

\section{Mercado dos equipamentos médico-hospitalares}

Os equipamentos médico-hospitalares são essenciais para o diagnóstico, tratamento, prevenção e reabilitação da saúde, viabilizando a oferta de serviços de qualidade à vida do usuário, atentando às exigências da ANVISA no que diz respeito à qualidade e segurança ${ }^{5}$.

As indústrias que compõem o setor de materiais possuem um elevado grau de conhecimento científico e inovação tecnológica, garantindo o desenvolvimento e aperfeiçoamento de produtos e, dessa forma, possuem significativa importância no suprimento de artigos tecnológicos voltados à saúde.

Os Estados Unidos concentra mais da metade $(56,0 \%)$ dos produtores de dispositivos médicos. A Europa e o Japão também têm participação importante no mercado, ambos com 18,0\% dos fabricantes, gerando um ambiente altamente dinâmico e 
competitivo, o que exige das empresas investimento na inovação ${ }^{8}$. Nesse alinhamento reflexivo, as empresas brasileiras se encontram em maior nível de dificuldade, pois apresentam menos incentivo ao desenvolvimento tecnológico quando comparadas com as grandes empresas estrangeiras do setor. Esses fatores interferem no valor do produto, pois o material estrangeiro absorve grandes taxas tributárias, além de estar vinculado a fatores como a política da marca, concorrência e transparência no mercado ${ }^{8}$. Entende-se, frente a essa constatação, como o processo de aquisição de equipamentos pelos serviços de saúde brasileiros é burocrático e de alto custo, devido à ausência de política de preço que rege essa modalidade. Trata-se, portanto, de uma área complexa, pois envolvem recursos financeiros relativamente altos, como também, interesses envolvendo a indústria e os participantes do serviço de saúde.

O mercado de equipamentos médicos mantem contato direto com as instituições de saúde públicas e privadas. Todas as instituições necessitam de materiais médicos para suprir as demandas de trabalho e, nesse contexto, é importante ressaltar que o profissional da organização pública e privada que está à frente das negociações, tem como principais atribuições, no desempenho do trabalho, a imparcialidade, ética e responsabilidade, visando sempre fazer mais e melhor com os recursos disponíveis.

Cabe a todos os envolvidos na cadeia de gestão de materiais uma ampla discussão sobre a perspectiva do preço dos equipamentos médicos que tanto impacta no custo dos setores da saúde. Também é necessário aprimorar o conhecimento sobre o tema, no cenário nacional e mundial, analisar o setor sob a ótica da concorrência, regulação, carga tributária, porte das instituições e preferência dos profissionais ${ }^{14}$.

\section{Gerenciamento do equipamento médico-hospitalar}

A gestão de equipamento médico-hospitalar é uma área que atua na tomada de decisões referentes ao custo e à aquisição de insumo, visando atender às diversas complexidades que o ambiente hospitalar enfrenta diariamente como a necessidade tecnológica, as exigências legais e as restrições no orçamento econômico ${ }^{12}$.

O gerenciamento dos equipamentos em uma organização hospitalar inicia desde a previsão de aquisição, até o seu uso, caracterizando, dessa forma, um processo muito minucioso. Nesse entendimento, medidas de planejamento com estudos precisos de todo processo devem ser introduzidas com o objetivo de obter um adequado equilíbrio financeiro e controle de manutenção ${ }^{11,14}$.

Já é sabido que as instituições absorvem grandes custos e uma maneira de reduzir essa perda é através do adequado processo de compra. Dessa forma, uma pesquisa desenvolvida com profissionais sobre a influência da gestão de equipamento médico-hospitalar no processo de trabalho aponta para a burocracia no processo de compra nas instituições; falta de qualidade de alguns materiais; e ausência de autonomia e envolvimento profissional na gestão de material médico hospitalar ${ }^{15}$.

Nas instituições privadas, a compra é realizada de forma direta. A própria instituição mantém contato direto com fornecedores, fazendo com que o processo seja menos burocrático e mais rápido.

Em relação aos setores públicos, os processos de compras exigem cumprimento da legislação do estatuto das compras públicas da Lei no 8.666 de 1993, que introduz a modalidade de compra via pregão, regulando todos os procedimentos do processo de aquisição de insumos, com o objetivo de buscar a proposta mais vantajosa dos fornecedores, observando os princípios da lei, onde o critério de menor preço sempre prevalecerá15,16.

Contudo, para atingir a redução dos custos e melhorias na prestação de serviços no processo de aquisição de equipamentos médico-hospitalares, deve-se levar em consideração, além do valor/preço, exigências mínimas de segurança, efetividade, cumprimento das especificações contratadas no seu registro junto a ANVISA, o uso 
racional e até mesmo, considerações sobre a satisfação dos usuários e qualidade do material oferecido ${ }^{17}$.

Todavia, cabe ressaltar que a qualidade do produto adquirido está ligada diretamente à relação de análise custo-benefício, sobressaindo o fator preço. Nesse sentido, um estudo sobre a percepção dos trabalhadores de enfermagem em relação às causas do desperdício de materiais chama atenção para a má qualidade dos materiais adquiridos e ao aumento do desperdício do mesmo ${ }^{18}$. Assim, a avaliação do equipamento deve ocorrer antes do processo de compra para impedir a entrada de qualidade inferior ao modelo do material desejado e refletir, dessa forma, na assistência prestada ${ }^{19}$.

Atrelada a isso, outra medida, a fim de reduzir custos e garantir a qualidade dos equipamentos, é a implantação de comissões técnicas devidamente estruturadas dentro da instituição, também chamadas de grupos de profissionais ou câmaras técnicas, composta por profissionais da assistência e representantes técnicos das principais áreas. Alguns autores afirmam que esse modelo de gerenciamento auxilia no processo de compra, pois são os profissionais que detêm conhecimento acerca de procedimentos e garantia de aquisição de dispositivos adequados ${ }^{15,17}$. Para a ANVISA, essa comissão pode também contribuir no processo de padronização, recebendo solicitação de avaliação de artigos médico-hospitalares ${ }^{20}$.

No âmbito da saúde pública, a preocupação com o gerenciamento de produtos e serviços levou á implantação do Programa Nacional de Gestão de Custos. Este programa foi implantado por meio de um manual, lançado em 2006, com a função de promover e facilitar a gestão de custos nas instituições hospitalares, com implicações na tomada de decisão, visando melhorias e resultados na prestação de serviços ${ }^{21}$.

Além disso, a Política Nacional de Gestão de Tecnologias em Saúde (PNGTS), resultado da Portaria $\mathrm{n}^{\circ}$ 2.510/GM, de 19 de dezembro de 2005, formada por diferentes segmentos da sociedade e coordenada pelo Ministério da Saúde, é o instrumento norteador para os atores envolvidos na gestão dos processos de avaliação, incorporação, difusão, gerenciamento da utilização e retirada de tecnologias no SUS. Atendendo às prerrogativas da PNGTS, as Diretrizes Metodológicas de Elaboração de Estudos para Avaliação de Equipamentos Médicos Assistenciais, foram desenvolvidas com o propósito de nortear a análise de técnicos e gestores interessados na avaliação de equipamentos médicos, estruturada por meio de domínios Clínico, Admissibilidade, Técnico, Operacional, Econômico e de Inovação ${ }^{22}$.

Como citado anteriormente, o desperdício de equipamentos relacionado ao uso inadequado e a deficiente forma de gerenciamento nas instituições de saúde favorecem e contribuem para a demasiada elevação de custos. Essa abordagem tem sido discutida e analisada conforme observado no estudo realizado em um hospital universitário, onde os maiores desperdícios identificados estão relacionados aos materiais de consumo, seguidos pelo desperdício de estrutura física ${ }^{23}$.

O desperdício de material é mais comum e frequentemente está associado à falta de capacitação e conscientização dos atuantes na assistência, sobre adequado uso e manuseio. Isso é observado principalmente no setor público, onde muitas instituições não apresentam sistema de informação, a fim de interligar a gestão de material ao estoque $^{22,24}$.

A informatização dos setores é uma medida que garante controle mais preciso do banco de dados de equipamento em estoque, em relação à entrada e saída de cada item, na criação de um histórico de peças consumidas por período de tempo e auxílio ao planejamento de aquisição de peças, diminuindo, assim, a incidência de falta de materiais e garantindo precisão no trabalho $0^{3,23}$.

Nesse contexto, outros prejuízos observados são em relação à falta de manutenção de equipamentos como, também, relacionados à estrutura física. A falta de manutenção nas válvulas de gases, por exemplo, pode custar à instituição valores monetários muito altos, levando à reflexão da importância de um controle institucional sistematizado ${ }^{23}$. 


\section{Estratégias de gerenciamento}

O processo de gestão dos equipamentos médico-hospitalares nas organizações de saúde inicia quando é observada a necessidade de um produto no setor ou na unidade. Quando o equipamento encontra-se padronizado, ocorre verificação de disponibilidade ou necessidade de compra. Por outro lado, quando o produto não está padronizado na instituição, em alguns casos, é emitido um comunicado à comissão de avaliação de equipamento para definição de características e da necessidade de compra. Caso contrário, o descritivo do equipamento é avaliado e substituído por outro produto similar, devidamente cadastrado na instituição ${ }^{17}$.

Dentro da cadeia de gerenciamento do equipamento médico-hospitalar, a compra é apenas uma fase desse processo, compreendendo, também, o armazenamento, a distribuição e o planejamento do controle de estoque. Cada material do estoque possui um valor e uma importância. Por isso, estratégias de análise para gerenciamento de estoque de material têm sido empregadas nas organizações de saúde com a finalidade de auxiliar na escolha e prioridade do material a ser reposto, otimizando-se os recursos financeiros ${ }^{25}$.

O setor de estoque sofre constantemente com pressões vinculadas à manutenção do estoque elevado, visando suprir a qualquer momento as necessidades de cada setor. Todavia, a reposição do estoque é indicada sempre quando a quantidade de material estiver insuficiente para atender as necessidades do prazo de abastecimento, período compreendido entre a solicitação e a chegada do pedido. A quantidade a ser adquirida deve ser a mínima suficiente, visando sempre a economia ${ }^{26}$.

$\mathrm{O}$ custeio baseado em atividades, conhecido como ABC - activity based costing - é um exemplo de ferramenta e estratégia utilizada no auxílio da gestão de equipamentos. É um procedimento que visa separar os materiais em grupos com características semelhantes, em função de seus valores e consumos, a fim de proceder a um processo de gestão apropriado a cada grupo. Essa metodologia é importante e muitas instituições de saúde utilizam esse instrumento, buscando maior controle na organização dos serviços ${ }^{27,28}$.

A classificação em grupo no método $A B C$ auxilia na organização e manutenção do estoque, sintetizado da seguinte maneira: grupo A - itens que geram maiores gastos, não em quantitativo unitário, mas pelo motivo de alto consumo financeiro; grupo B- corresponde aos itens em situação intermediária, representando um valor menos expressivo e, grupo C - materiais de baixo custo financeiro, mas de consumo elevado ${ }^{19}$.

O estabelecimento de grupos de equipamentos fornece subsídios para controle mais preciso. No entanto, é importante adotar acompanhamento para quantificar e qualificar os resultados.

Contudo, um fator a se considerar é a difícil tarefa de eleger critérios para indicar a importância de um item em relação a outro e uma maneira de auxiliar nessa questão e também no processo de aquisição de equipamento é a chamada análise de custo-benefício.

A análise de custo-benefício é uma área da economia que tem como objetivo identificar e avaliar todos os custos e benefícios associados a diferentes alternativas, e, assim, determinar qual a alternativa que maximiza a diferença entre benefícios e custos. Estes dados são expressos em termos quantificáveis e constituem um instrumento importante na tomada de decisão acerca dos investimentos e da avaliação de escolha de equipamentos a serem padronizados nas instituições. Entretanto, quantificar os benefícios não é uma tarefa fácil e caracteriza uma das dificuldades de adesão a esse estudo/análise, exigindo da equipe de compras e comissões técnicas muito estudo de avaliação ${ }^{22}$.

Países como Austrália, Canadá, Suécia e Reino Unido, com sistemas públicos de saúde, possuem agências de avaliação de tecnologias de saúde, que utilizam critérios rígidos para a tomada de decisão quanto à absorção de novos procedimentos, equipamentos e insumos ${ }^{10}$. 
No Brasil, a Lei n ${ }^{\circ} 12.041$ de 28 de Abril de 2011 estabelece, a respeito da assistência terapêutica e da aquisição de tecnologia em saúde, por meio da incorporação, a exclusão ou a alteração pelo SUS de novos medicamentos, materiais e procedimentos. Estabelece também a constituição, a alteração de protocolo clínico ou de diretriz terapêutica, baseada na tomada de decisão em evidências, levando em consideração aspectos como eficácia, efetividade e segurança da nova tecnologia, além da avaliação econômica, comparativa dos custos e benefícios em relação às tecnologias já existentes ${ }^{10,29}$.

Para dar suporte à tomada de decisões governamentais, o método de análise custo-benefício é o que cumpre melhor as exigências, na estimação dos benefícios e dos custos, pois leva em conta uma ampla gama de impactos e não somente o lucro ${ }^{24}$.

A função de planejar e controlar os equipamentos médico-hospitalares corresponde à área de gestão, a qual está ligada diretamente com os resultados financeiros da instituição. Por isso, cabe a todos envolvidos, em toda a cadeia de gerenciamento de equipamentos, trabalhar em prol da qualidade e produtividade, visando à redução de custos.

\section{Conclusão}

O trabalho teve como objetivo realizar uma reflexão acerca da temática gestão de equipamentos médico-hospitalares.

O aumento da demanda na área da saúde exige que instituições hospitalares acompanhem a evolução do mercado e invistam em infraestrutura e eficiência do atendimento. $\mathrm{O}$ trabalho demonstra vários indícios de viabilidade e dos aspectos favoráveis da utilização de processos de gestão, específicos em toda a cadeia de gerenciamento de equipamento nas instituições de saúde, como: estudos para aquisição de materiais, adoções de medidas de controle de estoque e participação ativa dos profissionais para combate de custos excessivos e desnecessários.

Contudo, deve-se ter em mente que a escolha do equipamento deve ser precedida de um estudo baseado no critério do custo-benefício, viabilizand o o processo de compra.

É importante que os serviços de saúde aprimorem os sistemas de gerenciamento de equipamentos, com preços de mercado de equipamentos de consumo, a fim de garantir uma assistência contínua e de qualidade a um menor custo e, ainda, assegurar a quantidade e qualidade dos equipamentos necessários para a realização do trabalho.

Uma medida que viabiliza a gestão é a aquisição de material de boa qualidade, com bom custo e benefício, o que acontece por meio da autonomia e com a participação ativa dos profissionais que atuam na assistência, na escolha do equipamento e no controle de custos.

A avaliação e as análises desenvolvidas nesse estudo são válidas por sua contribuição ao entendimento das dificuldades e das limitações existentes no processo de gerenciamento do equipamento médico-hospitalar.

\section{Referências}

1. Campos DF, Marques ICP. Custeamento ABC numa organização hospitalar privada: um estudo comparativo do custo de cirurgias eletivas com os valores reembolsados pelos planos de saúde. In: XXXV Encontro da ANPAD Associação Nacional de Pós-graduação e Pesquisa em Administração; 2011. Rio de Janeiro; 2011.

2. Kotler SS. Marketing estratégico para a área da saúde. Bookman; 2010. Definição do sistema de saúde e das escolhas conflitantes envolvidas.

3. Souza AA, Pereira ACC, Xavier AG, Xavier DO, Mendes ES. Logística hospitalar: um estudo de caso diagnóstico das dificuldades na gestão logística do setor de engenharia clínica. REAd. Rev. Eletronica Adm. 2013;12(1):1-14.

4. Freire RP, Pitassi C, Gonçalves AA, Schout D. Gestão de equipamentos médicos: o papel das práticas de qualidade em um hospital de excelência brasileiro. RAHIS. 2012;8(8):30-43.

5. Brasil. Agência Nacional de Vigilância Sanitária - ANVISA. Portaria nº 2.043, de 12 de dezembro de 1994 e Portaria SVS nº 686, de 27 de agosto de 1998. Conceitos técnicos, produtos para área da saúde. Inciso IV do Art. $3^{\circ}$, Decreto n 79.094, 
de 5 de janeiro de 1997 [citado em 2016 Fev 11]. Disponível em: http://portal.anvisa.gov.br/wps/content/Anvisa+Portal/ Anvisa/Inicio/Produtos+para+Saude

6. Brasil. Presidência da República. Ministério da Saúde Portaria n ${ }^{\circ} 2.915$, de 12 de dezembro de 2011. Institui a Rede Brasileira de Avaliação de Tecnologias em Saúde (REBRATS). 2011 [citado em 2016 Fev 17]. Disponível em: http://bvsms.saude. gov.br/bvs/saudelegis/gm/2011/prt2915_12_12_2011.html

7. Gil RB, Chaves LDP, Laus AM. Gerenciamento de recursos materiais com enfoque na queixa técnica. Rev. Eletr. Enf. 2015;17(1):100-7. http://dx.doi.org/10.5216/ree.v17i1.27544.

8. ANAHP. Observatório ANAHP. São Paulo; 2015 [citado em 2015 Fev 14]. Disponível em: http://www.anahp.com.br/files/ observatorio_anahp_2015_miolo_210x297_web.pdf

9. Reinhardt W Fo. Gestão de suprimentos e medicamentos. In: Vecina G No, Malik AM, editores. Gestão em saúde. Rio de Janeiro: Guanabara Koogan; 2011. p. 191-202.

10. Santos GMM, Carneiro LA. Materiais e medicamentos: vetores de custo no setor de saúde suplementar. IESS. 2013;0046:1-13.

11. Lopes LA, Dyniewicz AM, Kalinowski LC. Gerenciamento de materiais e custos hospitalares em UTI neonatal. Cogitare Enferm. 2010;15(2):278-85.

12. Amaral HCM. O processo de planejamento estratégico do Hospital Universitário da Universidade Federal de Santa Catarina: entre a intenção e a prática [dissertação]. Florianópolis: Universidade Federal de Santa Catarina, Centro Socioeconômico. Programa de Pós-graduação em Administração Universitária; 2013.

13. Marconi MA, Lakatos EM. Técnicas de pesquisa. 5. ed. São Paulo: Atlas; 2002.

14. Coelho FA, Nascimento JJ, SilvaJLG, Rodrigues JLK. A gestão de medicamentos e materiais médicos no resultado financeiro de um hospital público. In: The 4th International Congress on University-Industry Cooperation; 2012. Taubate; 2012.

15. Garcia SD, Haddad MCL, Dellaroza MSG, Costa DB, Miranda JM. Gestão de material médico-hospitalar e o processo de trabalho em um hospital público. Rev Bras Enferm. 2012;65(2):339-46. http://dx.doi.org/10.1590/S0034-71672012000200021.

16. Brasil. Presidência da República. Casa Civil. Subchefia para Assuntos Jurídicos Lei no 8.666 de Junho de 1993 Regulamenta o art. 37, inciso XXI, da Constituição Federal, institui normas para licitações e contratos da Administração Pública e dá outras providências. Portal da Legislação: Leis Ordinárias. Brasília; 2011 [citado em 2016 Fev 17]. Disponível em: http:// www.planalto.gov.br/CCIVIL_03/leis/L8666cons.htm

17. Reis LB, Barbosa IA, Soares LJF, et al. Análise das reprovações de materiais médico-hospitalares de um hospital de ensino. RAHIS. 2015;12(3):10-21.

18. Vaghetti HH, Roehrs M, Pires AC, Rodriguez C. Desperdício de materiais assistenciais na percepção de trabalhadores de enfermagem de um hospital universitário. Rev Enferm. 2011;19(3):369-74.

19. Dallora MELV, Forster AC. Gerenciamento de custos de material de consumo em um hospital de ensino. RAS. 2013;15(59):46-52.

20. Brasil. Agência Nacional de Vigilância Sanitária - ANVISA. Vigilância sanitária e licitações públicas. Brasília; 2016 [citado em 2016 Fev 9]. Disponível em: http://portal.anvisa.gov.br/wps/wcm/connect/fc9a4b00474591589989dd3fbc4c6735/ cartilha_licitacao.pdf?MOD=AJPERES

21. Brasil. Ministério da Saúde. Programa Nacional de Gestão de Custos: manual técnico de custos: conceitos e metodologias. Brasília; 2006 [citado em 2016 Fev 15]. Disponível em: http://portal.anvisa.gov.br/wps/wcm/connect/2cc0550047457fc48 b83df3fbc4c6735/Programa+Nacional+de+Gestao+de+Custos.pdf?MOD=AJPERES

22. Brasil. Ministério da Saúde. Secretaria de Ciência, Tecnologia e Insumos Estratégicos. Departamento de Ciência e Tecnologia. Diretrizes Metodológicas: estudos de avaliação econômica de tecnologias em saúde. Brasília; 2009. 145 p.

23. Castilho V, Castro LC, Couto AT, et al. Levantamento das principais fontes de desperdício de unidades assistenciais de um hospital universitário. Rev Esc Enferm USP. 2011;45:1613-20. PMid:22282070. http://dx.doi.org/10.1590/S0080-62342011000700012.

24. Campos CV, Santos LGS. A percepção do enfermeiro sobre o seu papel no gerenciamento de custos hospitalares. Rev. Min. Enferm. 2008;12(2):249-56.

25. Vecina G No, Reinhardt W Fo. Gestão de Recursos Materiais e de Medicamentos. São Paulo: Faculdade de Saúde Pública da Universidade de São Paulo; 1998. Série Saúde \& Cidadania para Gestores Municipais de Serviços de Saúde, 12.

26. Meaulo MP, Pensutti M. A gestão de estoques em ambientes hospitalares - analisando a utilização de ferramentas de gestão de materiais em ambientes hospitalares públicos e filantrópicos no município de Santa Bárbara D’Oeste. VIII Convibra Administração - Congresso Virtual Brasileiro de Administração. São Paulo; 2011. p. 1-12 [citado em 2016 Fev 9]. Disponível em: www.convibra.com.br

27. Duarte NCM, Bitar JPS, Miglioli JP, et al. Gestão de compras em um hospital de ensino terciário: um estudo de caso. Rev Med. 2015;48(1):48-56.

28. Bonacim CAG, Araujo AMP. Gestão de custos aplicada a hospitais universitários públicos: a experiência do Hospital das Clínicas da Faculdade de Medicina de Ribeirão Preto da USP. Rev. Adm. Pública. 2010;44(4):903-31. http://dx.doi. org/10.1590/S0034-76122010000400007.

29. Brasil. Presidência da República. Casa Civil. Subchefia para Assuntos Jurídicos. Lei n ${ }^{\circ} 12.401$, de 28 de abril de 2011 . Altera a Lei no 8.080, de 19 de setembro de 1990, para dispor sobre a assistência terapêutica e a incorporação de tecnologia em saúde no âmbito do Sistema Único de Saúde - SUS Portal da Legislação: Leis Ordinárias. Brasília; 2011 [citado em 2016 Fev 916]. Disponível em: http://www.planalto.gov.br/ccivil_03/_Ato2011-2014/2011/Lei/L12401.htm

\section{Contribuição dos autores}

Lisiane Marcolin de Almeida: participou da elaboração e planejamento da redação do artigo com contribuições intelectuais. Helena Terezinha Hubert Silva: orientou as etapas do trabalho, e participou da revisão e redação do artigo. 